\title{
Microwave detectors based on the spin-torque diode effect
}

\author{
O.V. Prokopenko \\ Taras Shevchenko National University of Kyiv, 64/13 Volodymyrska Str., Kyiv 01601, Ukraine \\ E-mail: oleksandr.prokopenko@gmail.com
}

A.N. Slavin

Oakland University, 2200 North Squirrel Rd., Rochester MI 48309, U.S.A.

E-mail: slavin@oakland.edu

Received December 21, 2014, published online March 23, 2015

\begin{abstract}
The spin-transfer torque (STT) effect provides a new method of manipulation of magnetization in nanoscale objects. The STT effect manifests itself as a transfer of spin angular momentum between the parallel magnetic layers separated by a nonmagnetic spacer and traversed by a dc electric current. The transfer of the spin angular momentum from one layer to another could result in the excitation of the microwave-frequency magnetization dynamics in one of the magnetic layers. On the other hand, when a magnetization dynamics is excited in a magnetic layered structure by an external microwave signal both the structure electrical resistance and current through the structure will acquire microwave components resulting in the appearance of a rectified dc voltage on the magnetic structure. This "spin-torque diode effect" can be used for the development of ultra-sensitive spintorque microwave detectors (STMD). Below we present a brief review of our recent work on the general properties of STMDs, analyze the performance of the "resonance-type" and "threshold-type STMD" and consider the possible applications for such microwave detectors.
\end{abstract}

PACS: 85.75.-d Magnetoelectronics: devices exploiting spin polarized transport or integrated magnetic fields; 75.76.+j Spin transport effects;

07.57.Kp Bolometers; infrared, submillimeter wave, microwave, and radiowave receivers and detectors.

Keywords: spin-transfer torque, spin-torque diode, spin-torque microwave detector, magnetization dynamics.

\section{Introduction}

This paper describes spin-torque magnetic detectors a novel type of nano-scale devices based on the spin-torque diode effect and relevant for the emerging field of applied spintronics.

The spin-transfer-torque (STT) effect in magnetic multilayers was theoretically predicted in [1,2] and experimentally observed in [3-13]. It provides a new method of manipulation of the magnetization direction in nanomagnetic systems [14]: magnetization switching [3,4], generation of microwave oscillations under the action of a dc electric current [5-11] and the spin torque diode effect [12,13], which can then be used for the development of practical microwave detectors, so called spin-torque microwave detectors (STMD), and also for quantitatively measuring STT $[15,16]$.

The spin torque diode effect is a rectification effect of the input microwave current $I_{R F}(t)$ in a magnetoresistive junction, which is commonly observed in traditional regime of operation of an STMD, when the frequency $f_{S}$ of the current $I_{R F}(t)=I_{R F} \sin \left(2 \pi f_{s} t\right)$ is close to the ferromagnetic resonance (FMR) frequency $f_{0}$ of the junction. In this case the induced resonance oscillations of the junction resistance $R(t)$ can mix with the oscillations of the input microwave current $I_{R F}(t)$ and produce a sufficiently large output de voltage $U_{D C}=\left\langle I_{R F}(t) R(t)\right\rangle$ across the junction (here $\langle\ldots\rangle$ denotes averaging over the period of oscillations $1 / f_{s}$ ).

There are two different dynamical regime of STMD operation: the traditional or so-called IP-regime and the so-called OOP-regime. In the traditional in-plane (IP) regime of operation of an STMD $[12,13,17]$ the STT excites a small-angle in-plane magnetization precession about the equilibrium direction of magnetization in a free layer (FL) of a magnetic tunnel junction (MTJ) (see the red dashed curve in Fig. 1). In contrast to this well-known regime of STMD operation, below we also consider a 
different regime of operation of an STMD, namely OOPregime, based on the excitation of large-angle out-ofplane (OOP) magnetization precession under the action of an input microwave current $I_{R F}(t)$ (see the blue dashed curve in Fig. 1). We analyze the performance of an STMD in these both regimes and consider typical applications of such detectors.

\section{Fundamental properties of an STMD}

The operation of an STMD employing an MTJ is based on two related phenomena: the effect of tunneling magnetoresistance (TMR) and the above mentioned STT effect. The phenomenon of TMR exists because of the dependence of the resistance $R(\beta)$ of a MTJ on the angle $\beta$ between the magnetizations of the two ("free" and "pinned") magnetic layers of the junction. For symmetric unbiased junctions $R(\beta)$ can be written as [17]

$$
R(\beta)=\frac{R_{\perp}}{1+\eta^{2} \cos \beta},
$$

where $R_{\perp}$ is the junction's resistance in the perpendicular $(\beta=\pi / 2)$ magnetic state and $\eta$ is the dimensionless spinpolarization efficiency. The conventional TMR ratio for such system can be calculated as $\mathrm{TMR}=2 \eta^{2} /\left(1-\eta^{2}\right)$. Typical values of $\eta$ are about $0.5-0.7$, whereas $R_{\perp}$ can be estimated as $R_{\perp}=R A / S$, where $R A \sim 1-10 \Omega \cdot \mu \mathrm{m}^{2}$ is the resistance-area product of the MTJ and $S$ is its area. For typical sub-micron sizes of the junction, $R_{\perp}$ is much higher than the characteristic impedance $Z=50 \Omega$ of standard microwave transmission lines.

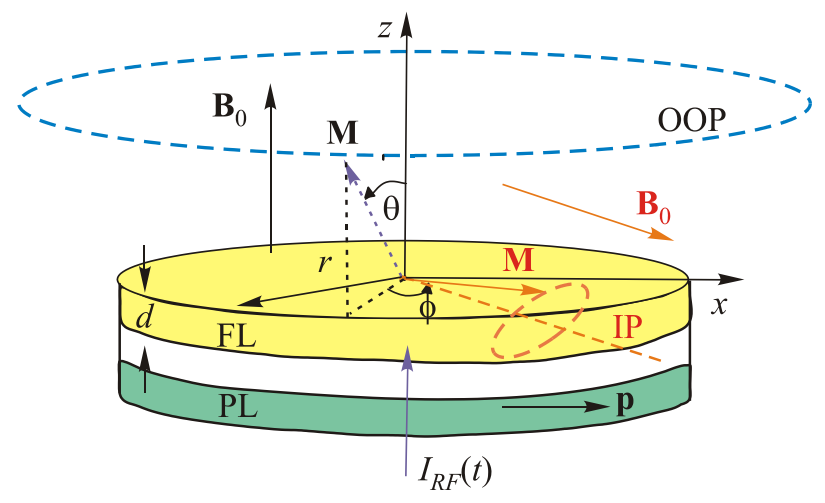

Fig. 1. (Color online) The model of an STMD: circular nanopillar of radius $r$ consists of the free magnetic layer (FL) of thickness $d$ and the pinned magnetic layer (PL). Under the action of microwave current $I_{F R}(t)$, the magnetization vector $\mathbf{M}$ (shown by a black or red arrow) is precessing along small-angle in-plane (red dashed curve, IP-regime) or large-angle out-of-plane (blue dashed curve, OOP-regime) trajectory about the direction of the bias magnetic field $\mathbf{B}_{0}$ (shown by a red or black arrow), $\mathbf{p}$ is the unit vector in the direction of the magnetization of the PL.
The dynamics of the magnetization $\mathbf{M}=\mathbf{M}(\mathbf{r}, t)$ in the FL of an STMD under the action of an input microwave current $I(t)$ is governed by the Landau-Lifshits-GilbertSlonczewski (LLGS) equation

$$
\begin{aligned}
\frac{\partial \mathbf{M}}{\partial t}= & \gamma\left[\mathbf{B}_{\mathrm{eff}} \times \mathbf{M}\right]+\frac{\alpha}{M_{S}}\left[\mathbf{M} \times \frac{\partial \mathbf{M}}{\partial t}\right]+ \\
& +\frac{\sigma(\beta) I(t)}{M_{S}}[\mathbf{M} \times[\mathbf{M} \times \mathbf{p}]],
\end{aligned}
$$

where $\gamma \approx 2 \pi \cdot 28 \mathrm{GHz} / \mathrm{T}$ is the modulus of the gyromagnetic ratio for electron spin, $\mathbf{B}_{\text {eff }}$ is the induction of the effective magnetic field acting on the magnetization $\mathbf{M}, \alpha$ is the dimensionless Gilbert damping constant, $M_{s}=|\mathbf{M}|$ is the saturation magnetization of the FL, $\mathbf{p}$ is the unit vector in the direction of the magnetization of the PL, and $\sigma(\beta)$ is the current-torque proportionality coefficient that is equal to

$$
\sigma(\beta)=\frac{\sigma_{\perp}}{1+\eta^{2} \cos \beta}, \quad \sigma_{\perp}=\frac{\gamma \hbar}{2 e} \frac{\eta}{M_{s} V},
$$

$\hbar$ is the reduced Planck constant, $e$ is the modulus of the electron charge, $V=S d$ is the volume of the FL ( $d$ is its thickness).

The output dc voltage $U_{D C}$ across the MTJ induced by an input microwave current $I(t)$ can be found as

$$
U_{D C}=\langle I(t) R(\beta(t))\rangle,
$$

where $\langle\ldots\rangle$ denotes averaging over the period of oscillations on input current, $R(\beta)$ is given by Eq. (1) and $\beta \equiv \beta(t)=\arccos \left(\mathbf{M} \cdot \mathbf{p} / M_{S}\right)$ depends on the solution $\mathbf{M}$ of the LLGS equation (2).

\section{Resonance-type STMD}

\subsection{General properties of a resonance-type STMD}

In the traditional regime of operation of an STMD the STT excites a small-angle in-plane magnetization precession about the equilibrium direction of the magnetization in the FL of the magnetic junction (the typical trajectory of the magnetization precession is shown by a red dashed curve in Fig. 1). The STMD working in the traditional IPregime has the following properties $[12,13,17]$.

(1) The STMD operates as a frequency-selective microwave detector with a resonance frequency that is close to the frequency of the ferromagnetic resonance (FMR) $\omega_{0}=2 \pi f_{0}$ of the FL.

(2) The range of operation frequencies of the detector is of the order of the FMR linewidth $\Gamma$.

(3) The output de voltage $U_{D C}$ of the STMD is proportional to the input microwave power $P_{R F}=I_{R F}^{2} R_{0} / 2$ ( $R_{0}=R\left(\beta_{0}\right)$ is the equilibrium MTJ resistance), so the 
STMD operates as a resonance-type quadratic microwave detector:

$$
U_{D C}=\varepsilon_{\mathrm{res}} P_{R F} \frac{\Gamma^{2}}{\Gamma^{2}+\left(\omega_{s}-\omega_{0}\right)^{2}},
$$

where

$$
\varepsilon_{\mathrm{res}}=\frac{\gamma \hbar}{4 e} \frac{\eta^{3}}{M_{s} V \Gamma} Q\left(\beta_{0}\right)
$$

is the resonance (at $\omega_{s}=\omega_{0}$ ) volt-watt sensitivity of the STMD (see [17]). Here $Q\left(\beta_{0}\right)$ is the geometrical factor that depends on the angle $\beta_{0}$ between the directions of the equilibrium magnetization in the FL and PL of the MTJ. For an in-plane magnetized MTJ we have:

$$
Q\left(\beta_{0}\right)=\frac{\sin ^{2} \beta_{0}}{\left(1+\eta^{2} \cos \beta_{0}\right)^{2}} ;
$$

(4) The STMD resonance sensitivity $\varepsilon_{\text {res }}$ strongly depends on the equilibrium angle $\beta_{0}$ between the magnetization directions of the FL and PL (see Eq. (7)).

The resonance sensitivity of a traditional STMD $\varepsilon_{\text {res }}=U_{D C} / P_{R F}$ is predicted to be approximately $10^{4} \mathrm{~V} / \mathrm{W}$ (see [17]), while the best experimental value achieved to date is $\varepsilon_{\text {res }} \approx 300 \mathrm{~V} / \mathrm{W}$ for an unbiased STMD [13]. However, $\varepsilon_{\text {res }} \approx 12000 \mathrm{~V} / \mathrm{W}$ has been recently obtained for an STMD biased by a dc current [18].

\subsection{Theoretical description of noise properties of a resonance-type STMD}

It is well-known that the operation and the minimum detectable power of any microwave detectors are limited by noise. Below we present a theoretical description of the noise properties of a passive (no dc bias current) resonance-type STMD using the model developed in [17] with additional terms describing the influence of thermal fluctuations. The results presented below are expanded and are complementary to the results presented in $[19,20]$.

We used the following assumptions.

(1) We use the macrospin approximation for the magnetization dynamics in the FL of an STMD.

(2) We neglect any possible fluctuations or oscillations of magnetization in the PL.

(3) We consider unbiased STMD, i.e., the current flowing through STMD has only a microwave component $I_{R F}(t)$.

(4) The amplitude of the detected current $I_{R F}$, as well as the temperature of the system $T$, are assumed to be sufficiently small to guarantee a linear response of the magnetization to the fluctuations of current and thermal fluctuations.

(5) We consider the case when the magnetic damping of the STMD is sufficiently low $\Gamma / 2 \pi<<f_{0}$.
(6) We also assume that the frequency interval of the detection $\Delta f$ (which is inversely proportional to the measurement time) is much smaller than the FMR linewidth $\Gamma / 2 \pi$ in the FL, $\Delta f<<\Gamma / 2 \pi$.

(7) Finally, we assume that the effective noise temperature $T$ for all the noise sources considered below is the same.

There are several possible sources of noise in an STMD. Moreover, since the operation of an STMD is nonlinear, the noise is, in general, not additive, and can depend on the amplitude and frequency of the detected signal. Among all the possible noise sources we shall consider only the following.

a) Low-frequency Johnson-Nyquist (JN) noise, i.e., low-frequency voltage fluctuations $U_{N}(t)$ associated with the equilibrium electrical resistance $R_{0}$ of the STMD. This type of noise is additive and is independent of the magnetization dynamics.

b) High-frequency Johnson-Nyquist noise, that does not contribute to the output detected signal directly. However, a microwave noise current $I_{N}(t)$ can induce additional fluctuations of the magnetization direction in the FL and, therefore, additional fluctuations of the electrical resistance of an STMD. Being mixed with the detected current $I_{R F}(t)$, these resistance fluctuations will create nonadditive low-frequency noise in the output voltage.

c) Magnetic noise (MN), which is caused by the thermal fluctuations of the magnetization direction in the MTJ FL. It is modeled by the action of a random white Gaussian magnetic field $\mathbf{B}_{N}(t)$ and leads to the fluctuations of the electric resistance of the STMD. It is transformed into a low-frequency noise after mixing with the driving current $I_{R F}(t)$.

The other noise sources, such as shot noise and flicker noise, that might be important for STMDs biased by a dc current, are not considered here.

To calculate the noise spectrum in an STMD we shall, first, calculate the linear response of the magnetization of the FL to arbitrary microwave currents and magnetic fields. Then we shall calculate the corresponding variations of the electrical resistance of the STMD, i.e., calculate the electrical response of STMD to currents and fields. After that, using a simplified electrical scheme of an STMD, we shall calculate the STMD noise spectrum.

Dynamics of the magnetization $\mathbf{M}$ of the FL under the action of microwave current $I(t)=I_{R F}(t)+I_{N}(t)$ and noise magnetic field $\mathbf{B}_{N}(t)$ is described by the LLGS Eq. (2), where the effective magnetic field is a sum of an intrinsic effective magnetic field $\mathbf{B}_{\text {eff }}=\mathbf{B}_{\text {eff }}(\mathbf{M})$ and the noise field $\mathbf{B}_{N}(t)$. The intrinsic field $\mathbf{B}_{\text {eff }}$ includes the external bias magnetic field $\mathbf{B}_{0}$ and the demagnetization field.

We performed analysis for a "planar" STMD in which both FL and PL are magnetized in-plane. In this case, the eigen-frequency of the FL of the MTJ is $f_{0}=$ $=(\gamma / 2 \pi) \sqrt{B_{0}\left(B_{0}+\mu_{0} M_{s}\right)}$ and the damping rate has the 
form $\Gamma=\alpha \gamma\left(B_{0}+\mu_{0} M_{s} / 2\right)$, where $\mu_{0}$ is the vacuum magnetic permeability. The output STMD signal was calculated as a low-frequency part of the voltage $I(t) R(\beta(t))+U_{N}(t)$, where $R(\beta)$ is the MTJ magnetoresistance (1).

We assumed that noise signals produced by all the noise sources are independent Gaussian stochastic processes with uniform spectral densities

$$
\begin{gathered}
S\left(U_{N}\right)=S\left(I_{N}\right) R_{0}^{2}=2 k_{B} T R_{0}, \\
S\left(B_{N}\right)=2 \alpha k_{B} T / \gamma M_{S} V
\end{gathered}
$$

where $k_{B}$ is the Boltzmann constant. The detailed derivation of the noise spectrum of STMD is presented in [20].

We found that the noise of the output voltage $U_{D C}=\varepsilon_{\text {res }} P_{R F}$ has a characteristic spectral width of $\Gamma$ and, for a typical frequency bandwidth of measurement $\Delta f \ll \Gamma / 2 \pi$, can be considered as frequency-independent. The root mean square fluctuations $\Delta U_{D C}$ of STMD can be written in a simple form

$$
\Delta U_{D C}=U_{J N} \sqrt{1+\frac{U_{D C}}{U_{I M}}+\frac{U_{D C}}{U_{M N}}},
$$

where the three terms under the square root describe the influence of the three above mentioned noise sources, respectively, and

$$
U_{J N}=\sqrt{4 k_{B} T R_{0} \Delta f}, \quad U_{I M}=\frac{R_{0}}{4 \varepsilon_{\mathrm{res}}}, \quad U_{M N}=\frac{\gamma \hbar}{2 e} \frac{B_{0}}{\eta} .
$$

Note, that both the high-frequency JN and magnetic noise give nonadditive contributions to the output lowfrequency signal and, therefore, their influence increases with the input power (or the output voltage $U_{D C}$ ). $U_{I M}$ and $U_{M N}$ are the characteristic output voltages at which the influence of the corresponding noise source becomes comparable to the influence of the additive low-frequency JN noise. Non-additive noises can be ignored for small output signals $U_{D C} \ll U_{I M}, U_{M N}$.

For typical parameters (see e.g. $[13,17]$ ) of an MTJ nanopillar $\left(r=50 \mathrm{~nm}, d=1 \mathrm{~nm}, \eta_{2}=0.7, \beta_{0}=\pi / 4, R_{0}=\right.$ $=500 \Omega$, (giving RA $=5.29 \Omega \cdot \mu \mathrm{m}^{2}$ ), $\alpha=0.01, \mu_{0} M_{s}=$ $=0.8 \mathrm{~T}, B_{0}=38 \mathrm{mT}$, (giving $f_{0}=5 \mathrm{GHz}$ ) ) Eq. (6) gives the resonance STMD sensitivity in the passive regime $\varepsilon_{\text {res }} \approx 700 \mathrm{~V} / \mathrm{W}$, which is comparable to the sensitivity of Schottky diodes [13]. At room temperature $T=300 \mathrm{~K}$ and for the measurement bandwidth of $\Delta f=1 \mathrm{MHz}$, Eq. (10) gives the following estimations for the noise-induced voltages: $U_{J N}=2.88 \mu \mathrm{V}, U_{I M}=175.9 \mathrm{mV}, U_{M N}=3.14 \mu \mathrm{V}$.

It is clear, that the characteristic voltage $U_{I M}$ of the nonadditive high frequency $\mathrm{JN}$ noise is much larger than the voltages created by the low frequency JN noise $U_{J N}$, magnetic noise $U_{M N}$, and the typical dc voltage output $U_{D C}$ of the STMD. This means that the influence of the highfrequency JN noise in (9) can be completely ignored. Note, also that, in contrast with the other characteristic noise voltages, the voltage $U_{M N}$ caused by the magnetic noise is proportional to the bias magnetic field $B_{0}$, and, therefore, increases with the increase of the frequency of the input microwave signal.

Now, introducing the microwave powers $P_{R F}=$ $=U_{D C} / \varepsilon_{\text {res }}, P_{M N}=U_{M N} / \varepsilon_{\text {res }}$, and using (9), we can write a simple expression for the signal-to-noise ratio (SNR) of the STMD in terms of these characteristic powers:

$$
\mathrm{SNR}=\frac{U_{D C}}{\Delta U_{D C}}=\frac{P_{R F}}{P_{J N}} \sqrt{\frac{P_{M N}}{P_{M N}+P_{R F}}} .
$$

\subsection{The performance of a resonance-type STMD in the presence of thermal noise}

The simple analysis of Eqs. (9), (11) demonstrates that there are two distinct regimes of operation of the resonance STMD in the presence of thermal noise. We shall classify them by the type of noise that limits the minimum detectable power of STMD $P_{\min }$ (power corresponding to $\mathrm{SNR}=1$ ).

The first regime corresponds to the case of relatively high frequencies of the input microwave signal, when $P_{M N} \gg P_{R F}$ (for $P_{R F} \sim P_{\min }$ ). In this regime, similar to the conventional semiconductor diodes, the minimum detectable power is limited by the low-frequency JN noise, $P_{\min }=P_{J N}$, and the SNR of STMD is linearly proportional to the input microwave power $P_{R F}: \mathrm{SNR} \cong P_{R F} / P_{J N}$.

The second regime takes place in the opposite limiting case of relatively low input frequencies, when $P_{M N} \ll P_{R F}$. In this case the SNR of the STMD increases with $P_{R F}$ much slower than in conventional diodes, and is proportional to the square root of the input microwave power: $\mathrm{SNR} \cong \sqrt{P_{R F} / P_{\min }}$. The minimum detectable power $P_{\min }=P_{J N}^{2} / P_{M N}$ in this regime is limited by the magnetic noise in the FL of the MTJ.

The existence of two distinct regimes of the STMD operation is illustrated in Fig. 2, where two curves calculated from Eq. (11) show the STMD SNR as functions of the input power for signal frequencies $f_{1}=1 \mathrm{GHz}$ (dashed blue line) and $f_{2}=25 \mathrm{GHz}$ (red solid curve). It can be seen, that both curves (presented in logarithmic coordinates) demonstrate a clear change of slope from 1 to $1 / 2$ in the region, where the input power $P_{R F}$ is close to the characteristic power of the magnetic noise $P_{M N}$ (which increases with the increase of the input signal frequency). The minimum detectable power $P_{\min }$ (corresponding to SNR =1) in the highfrequency case is smaller than $P_{M N}$ and lies in the region of the linear dependence of SNR on $P_{R F}$ (solid red line in Fig. 2). The situation is opposite in the low frequency case (blue dashed curve in Fig. 2), when $P_{\min }>P_{M N}$ and lies in the region, where the slope of the SNR curve is equal to $1 / 2$. 


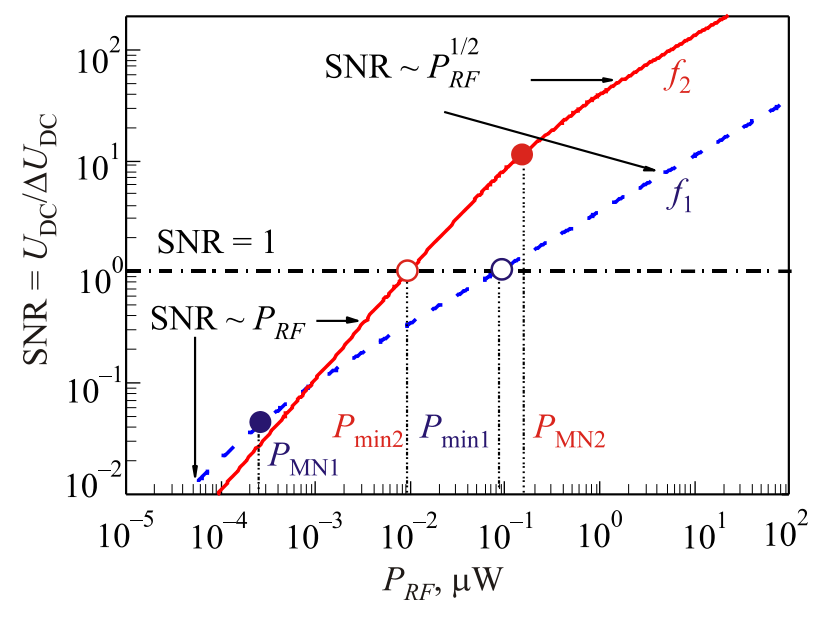

Fig. 2. (Color online) Typical dependence of the SNR of STMD on the input microwave power $P_{R F}$ calculated from Eq. (11) for two different frequencies of the input microwave signal: $f_{1}=$ $=1 \mathrm{GHz}$ (dashed blue line) and $f_{2}=25 \mathrm{GHz}$ (solid red line) [19]. $P_{\min }$ is the minimum detectable power of STMD (at SNR = 1) and $P_{M N}$ is the frequency-dependent characteristic power of magnetic noise.

The evolution of the characteristic powers $P_{M N}$ and $P_{\text {min }}$ with the increase of frequency of the input microwave signal $f$ is shown in Fig. 3. The curve $P_{M N}(f)$ separates the plane into the region, where magnetic noise is dominant (above the curve), and the region, where the STMD operation is limited by the JN noise (below the curve). It is, clear, that the smallest detectable power is achieved near the border of these two regimes.

When an STMD based on an MTJ nanopillar is used as a sensor of microwave radiation it is typically connected to a standard transmission line with the impedance of $Z=$ $=50 \Omega$. The minimum detectable microwave power delivered to a $50 \Omega$ transmission line can be written as [21]:

$$
P_{\min }[50 \Omega]=\frac{1}{4} \frac{\left(R_{0}+Z\right)^{2}}{Z R_{0}} P_{\min } .
$$

Using this expression and taking into account the size dependence of the STMD resistance $\left(R_{0} \propto 1 / r^{2}\right)$, it is possible to show that $P_{\min }[50 \Omega]$ has a clear minimum as a function of the nanopillar radius $r$. For instance, the optimum value of the nanopillar radius is $r_{\mathrm{opt}} \approx 100 \mathrm{~nm}$ for the input frequency $f=5 \mathrm{GHz}$ (see the inset in Fig. 3).

As one can see from Figs. 2 and 3, the STMD operating in the IP regime has a performance comparable to the performance of a conventional Schottky diode. Taking this into account we believe that such STMDs might be useful for the development of practical signal processing devices involving microwave detectors, such as sensors, frequency analyzers, etc.

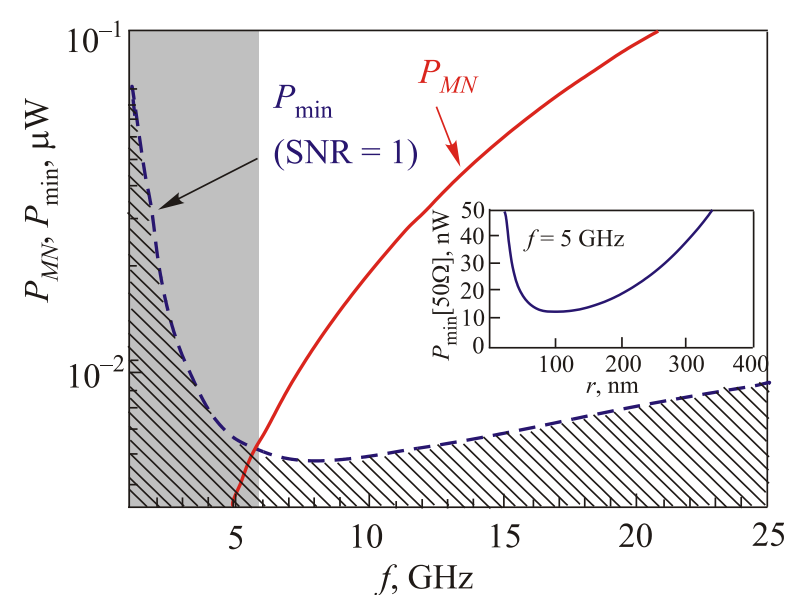

Fig. 3. (Color online) Characteristic power of magnetic noise (solid red line) and minimum detectable power $P_{\min }$ of an STMD (dashed blue line) as functions of the input microwave frequency $f$. The blue dashed area corresponds to undetectable signals $\left(P_{R F}<P_{\min }\right)$ and gray shaded area shows the low-frequency STMD regime, where the magnetic noise is dominant in the whole practical region $P_{R F}>P_{\text {min }}$. Inset: minimum detectible microwave power delivered to a $50 \Omega$ transmission line $P_{\min }[50 \Omega]$ for $f=5 \mathrm{GHz}$ as a function of the radius $r$ of the MTJ nanopillar.

\subsection{Influence of temperature on the performance of a resonance-type $S T M D$}

In the case of an STMD the temperature dependence of the detector's characteristics may be non-trivial since many STMD parameters besides the noise level (e.g., saturation magntetization and spin-polarization efficiency) also change with the temperature. In this section of the paper we analyze the influence of temperature $T$ on the performance of a passive STMD, namely, we studied the temperature dependences of the STMD sensitivity $\varepsilon_{\text {res }}$ [17], signal-to-noise ratio SNR, and minimum detectable microwave power $P_{\min }[50 \Omega][19,20]$. We believe that the developed formalism can be used for the optimization of the practical operational parameters of an STMD in a wide range of temperatures.

We assumed that the spin-polarization efficiency $\eta$ of the bias current flowing in an STMD depends on the temperature $T$ as

$$
\eta(T)=\eta(0)\left(1-\kappa T^{3 / 2}\right),
$$

where $\kappa$ is the temperature coefficient of spinpolarization, while the STMD resistance in a perpendicular magnetic state $R_{\perp}$ is determined by

$$
R_{\perp}(T)=R_{\perp}(0)(1-\chi T),
$$

where $\chi$ is the temperature coefficient of resistance $[22,23]$. We also used the following expression for the temperature dependence of the static magnetization $M_{s}(T)$ : 


$$
M_{s}(T)=M_{s}(0)\left(1-\frac{T}{T_{C}}\right)^{\tau}
$$

where $T_{C}$ is the Curie temperature and $\tau$ is the dimensionless parameter, typically having magnitude within the range from 0.1 and up to 0.5 [24].

In our calculations we used the following typical parameters of the $\mathrm{Ni}_{80} \mathrm{Fe}_{20} \mathrm{FL}$ in an MTJ nanopillar having composition $\mathrm{Co} / \mathrm{Al}_{2} \mathrm{O}_{3} / \mathrm{Ni}_{80} \mathrm{Fe}_{20}$ taken at room temperature $T=300 \mathrm{~K}$ (see e.g. [17,19,22,23]): temperature coefficient of spin polarization $\kappa=4 \cdot 10^{-5} \mathrm{~K}^{-3 / 2}$ [23], angle between equilibrium magnetization of the FL and PL $\beta_{0}=\pi / 2$, temperature coefficient of resistance $\chi=\chi_{0}=$ $=7.65 \cdot 10^{-4}, \mathrm{~K}^{-1}$ [23], equilibrium resistance of an STMD $R_{0}(300 \mathrm{~K})=1 \mathrm{k} \Omega$, the Curie temperature $T_{C}=822 \mathrm{~K}$ [25], power coefficient $\tau=0.4$. All the other parameters are presented in Sec. 2.3 (at $T=300 \mathrm{~K}$ ). We also assume that the power of the external microwave signal is $P_{R F}=1 \mu \mathrm{W}$.

The dependences of $\varepsilon_{\text {res }}(T)$ and $\operatorname{SNR}(T)$ on the temperature $T$ are shown in Fig. 4 and the temperature dependence of $P_{\min }[50 \Omega] T$ is shown in Fig. 5. One can see from Fig. 4, that for relatively high temperatures $(T>50 \mathrm{~K})$ the sensitivity $\varepsilon_{\mathrm{res}}(T)$ decreases monotonically with the increase of temperature $T$, mainly due to the temperature dependence of the spin polarization efficiency $\eta(T)$. At low temperatures $(T<50 \mathrm{~K})$, the sensitivity $\varepsilon_{\text {res }}(T)$ slightly increases with $T$. This behavior is explained by the reduction of the static magnetization $M_{S}(T)$, which, in this temperature interval, has a stronger temperature dependence than $\eta(T)$. The maximum of the curve $\varepsilon_{\text {res }}(T)$ corresponds to the temperature $T_{\varepsilon}$ at which the factor $\eta^{3}(T) / M_{s}(T) \Gamma(T)$ reaches its maximum value. Note, that in the case of $\beta_{0} \neq \pi / 2$ the reduction of the function $\varepsilon_{\text {res }}(T)$ with the decrease of temperature in the range $T<T_{\varepsilon}$ may become more pronounced. This means that

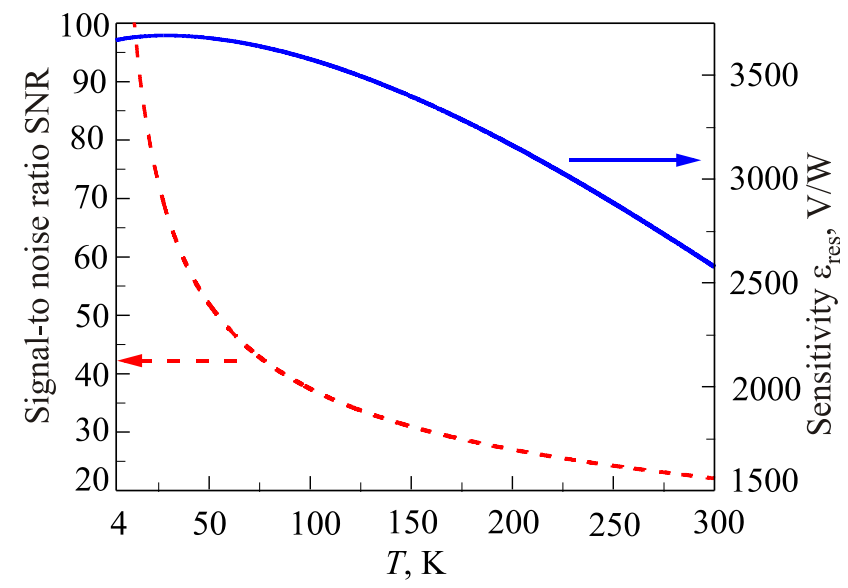

Fig. 4. Dependences of resonance volt-watt sensitivity $\varepsilon_{\text {res }}(T)$ (solid line) and signal-to-noise ratio $\operatorname{SNR}(T)$ (dashed line) on temperature $T$ for the STMD with typical parameters (see Sec. 2.4 for details).

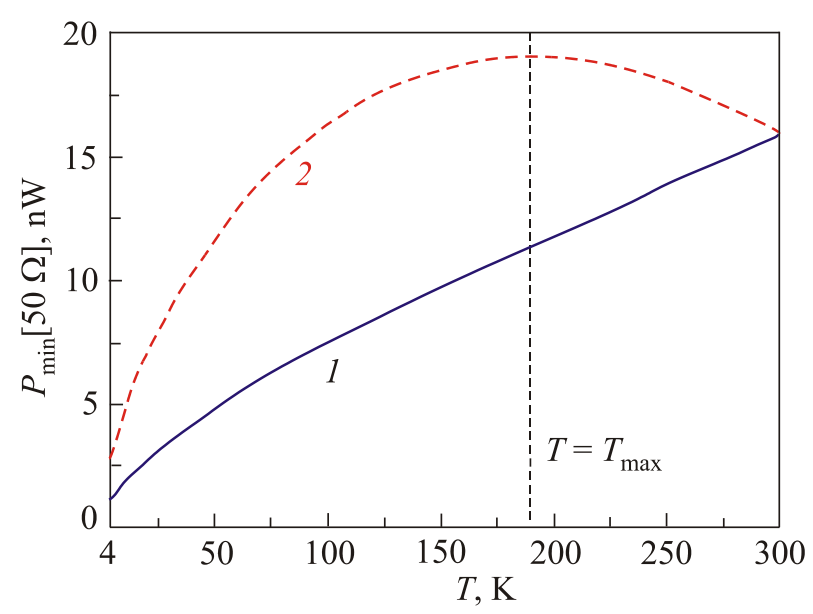

Fig. 5. Temperature dependences of a minimum detectable microwave power $P_{\min }[50 \Omega]$ for the STMD with temperature coefficient of resistance $\chi=\chi_{0}$ (curve 1 , solid line) and $\chi=2.5 \chi_{0}$ (curve 2, dashed line). All the other parameters are the same as in Fig. 4.

cooling an STMD to very low temperatures (of the order of $4 \mathrm{~K}$ ) may have an adverse effect in the form of the reduction of its sensitivity.

In contrast to the temperature dependence of $\varepsilon_{\text {res }}(T)$, the signal-to-noise ratio $\operatorname{SNR}(T)$ decreases monotonically with the increase of temperature (see dashed line in Fig. 4). This dependence is caused, mainly, by the rapid increase $(\sim \sqrt{T})$ of the Jonhson-Nyquist noise power $P_{J N}(T)$ with temperature.

The temperature dependence of the minimum detectable microwave power $P_{\min }[50 \Omega](T)$, shown in Fig. 5, is more complicated than the temperature dependencies $\varepsilon_{\text {res }}(T)$ and $\operatorname{SNR}(T)$. For some values of the detector's parameters the curve $P_{\min }[50 \Omega](T)$ may have a clear maximum at a certain temperature $T_{\max }$. For instance, this situation is realized for an STMD with a sufficiently high temperature coefficient of resistance $\chi$ (see curve 2 in Fig. 5).

The temperature dependence of $P_{\min }[50 \Omega](T)$ is determined by two main factors: by the temperature dependence of the "intrinsic" minimum detectable power $P_{\min }(T)$ (without taking into account the impedance mismatch effect), which can be obtained from the condition $\operatorname{SNR}(T)=1$, and the temperature dependence of the impedance mismatch coefficient between the detector and the transmission line. Since the $\operatorname{SNR}(T)$ decreases monotonically with the increase of the temperature, the "intrinsic" power $P_{\min }(T)$ also monotonically increases with $T$. For a sufficiently small temperature coefficient of resistance $\chi$, the temperature dependence of $P_{\min }[50 \Omega](T)$ is determined mainly by the "intrinsic" minimum power $P_{\min }(T)$ and has a monotonic shape (see curve 1 in Fig. 5).

On the other hand, if the temperature coefficient of resistance $\chi$ is large, the temperature dependence of the mismatch may become stronger than the dependence $P_{\min }(T)$. As a result, the minimum detectable power 
$P_{\min }[50 \Omega](T)$ decreases at high temperatures and reaches a maximum value at a certain finite temperature $T_{\max }$ (see curve 2 in Fig. 5). In this regime, cooling of a STMD improves its characteristics only in the low-temperature region $T<T_{\max }$.

\section{Threshold-type STMD}

In contrast to the well-known IP regime of the STMD operation, below we consider a different regime of operation of an STMD, based on the excitation of a large-angle out-of-plane (OOP) magnetization precession under the action of a sufficiently large (above threshold) input microwave current $I_{R F}(t)$. We show that this regime of the STMD operation is realized when the STMD is biased by the perpendicular magnetic field $\mathbf{B}_{0}=\mathbf{z} B_{0}$, which is smaller than the saturation magnetic field of the FL, i.e., $B_{0}<\mu_{0} M_{s}$.

\subsection{Theoretical description of the threshold-type STMD working in the OOP-regime}

We consider a simple model of an STMD, formed by a circular MTJ nano-pillar (see Fig. 1). The magnetization of the PL of the MTJ is assumed to be completely fixed and lie in the plane of the layer. The direction of the PL magnetization $\mathbf{p}=\mathbf{x}$ determines the axis of spin-polarization. The radius $r$ of the MTJ nano-pillar is assumed to be sufficiently small, so that the magnetization $\mathbf{M} \equiv \mathbf{M}(t)$ of the free FL is spatially-uniform and can be treated in the macrospin approximation. For simplicity, we neglect any in-plane anisotropy of the FL.

The dynamics of the magnetization vector $\mathbf{M}(t)=$ $=\mathbf{m}(t) M_{S}$ in the FL under the action of a microwave current $I(t)=I_{R F} \sin \left(\omega_{s} t\right)$ is governed by the LLGS Eq. 2 where $\mathbf{B}_{\text {eff }}=\left(B_{0}-\mu_{0} M_{z}\right) \mathbf{z}, M_{z}$ is the $z$-component of vector $\mathbf{M}$.

Using the spherical coordinate system one can express the normalized magnetization vector as $\mathbf{M} / M_{s}=\mathbf{x} \sin \theta \cos \varphi+$ $+\mathbf{y} \sin \theta \sin \varphi+\mathbf{z} \cos \theta$ and obtain the following equations for the polar $\theta$ and azimuthal $\varphi$ angles characterizing the position of the vector $M / M_{s}$ :

$$
\begin{gathered}
\frac{d \theta}{d t}=-\alpha \omega_{P} \sin \theta-\sigma I_{R F} \sin \left(\omega_{s} t\right) \cos \theta \cos \varphi, \\
\frac{d \varphi}{d t}=\omega_{P}+\sigma I_{R F} \sin \left(\omega_{s} t\right) \csc \theta \sin \varphi
\end{gathered}
$$

Here $\omega_{P} \equiv \omega_{P}(\theta)=\omega_{H}-\omega_{M} \cos \theta$ is the frequency of the magnetization precession in the OOP-regime, $\omega_{H}=\gamma B_{0}$, and $\omega_{M}=\gamma \mu_{0} M_{s}$. For simplicity, in Eqs. (16) we neglected small nonconservative terms $\left(\sim \alpha^{2}\right.$ and $\left.\sim \alpha I_{R F}\right)$ which have a negligible effect on the magnetization dynamics.

In the regime of a large-angle OOP precession, the magnetization of the FL performs precession around the $z$ axis along an approximately circular orbit, $\theta \approx$ const, $\varphi \approx \omega_{s} t+\psi$, where $\psi$ is the phase shift between the magnetization precession and the driving current. To analyze the conditions, under which the OOP regime is possible, one can average Eqs. (16) over the period of precession $2 \pi / \omega_{s}$, and obtain the averaged equations for the slow variables $\theta$ and $\psi$ (see Eqs. (11.39) in [20] for details).

The OOP regime of magnetization precession corresponds to a stationary solution of the averaged equations for slow variables $\theta=\theta_{s}=$ const, $\psi=\psi_{s}=$ const. In this case one can find the conditions defining the stationary value of the phase shift $\psi_{s}$ :

$$
\sin \psi_{s}=2 \alpha \frac{\omega_{P}}{\sigma_{\perp} I_{R F}} \tan \theta_{s}, \quad \cos \psi_{s}=2 \frac{\omega_{s}-\omega_{P}}{\sigma_{\perp} I_{R F}} \sin \theta_{s} .
$$

Eliminating $\psi_{s}$ from the above equations, one obtains the equation for $\theta_{s}$ :

$$
\left(\omega_{s}-\omega_{P}\right)^{2} \sin ^{2} \theta_{s}+\alpha^{2} \omega_{P}^{2} \tan ^{2} \theta_{s}=\frac{1}{4} \sigma_{\perp}^{2} I_{R F}^{2} .
$$

This equation for $\theta_{s}$ is a nonlinear equation, which, in a general case, can be only solved numerically. One can see that Eq. (18) has solutions only for microwave currents $I_{R F}$ larger than a certain threshold current $I_{\text {th }}$. At this threshold current $\omega_{P}\left(\theta_{s}\right) \approx \omega_{s}$, and the magnitude of the threshold microwave current $I_{\text {th }}\left(\omega_{s}\right)$ needed to excite the OOP precession is:

$$
I_{\mathrm{th}}\left(\omega_{s}\right)=2 \alpha \frac{\omega_{M}}{\sigma_{\perp}} \frac{\omega_{s}}{\omega_{H}-\omega_{s}} .
$$

The OOP regime will be stable if the following approximate conditions are fulfilled [26]:

$$
0<\cos \theta_{s}<\frac{\omega_{H}}{\omega_{M}}, \quad \omega_{s}<\omega_{H}
$$

The output voltage of an STMD working in the OOP regime can be found as [20]

$$
U_{D C} \approx w I_{\mathrm{th}}\left(\omega_{S}\right) R_{\perp},
$$

where $\quad w(a)=1 / \sqrt{1-a^{2}}\left(1-\sqrt{1-a^{2}} / a\right), \quad a=\eta^{2} \sin \theta$. It follows from Eq. (19), (21) that the output dc voltage of an STMD practically does not depend on the amplitude of the microwave current, provided that it is larger than the threshold value $I_{\text {th }}$.

\subsection{Performance of an $S T M D$ in the OOP regime}

Below we shall analyze the above presented analytical description for an STMD operating in the OOP regime, compare this analytical description with the results of numerical simulations of an STMD and, then, also compare the performance of the STMD in the IP and OOP regimes.

We consider the case of an STMD with the typical parameters specified in Sec. 2.2. We choose the magnitude of 
the external out-of-plane dc magnetic field to be $B_{0}=$ $=200 \mathrm{mT}$ for the STMD in the OOP regime, which corresponds to the maximum OOP precession frequency of $\omega_{H}=2 \pi \cdot 5.6 \mathrm{GHz}$. In the IP regime of operation, the STMD will be characterized by the equilibrium angle $\beta_{0}=\pi / 2$ between the equilibrium magnetization of the $\mathrm{FL}$ and the magnetization of the PL. Hence, the equilibrium resistance of the STMD in the IP regime is $R_{0}=$ $=R_{\perp}=1 \mathrm{k} \Omega$. We choose the magnitude of the external DC in-plane magnetic field as $B_{0}=14.1 \mathrm{mT}$ for the STMD in the IP regime, that in accordance with the expression for the FMR frequency $f_{0}=(\gamma / 2 \pi) \sqrt{B_{0}\left(B_{0}+\mu_{0} M_{s}\right)}$, gives $f_{0}=$ $=3 \mathrm{GHz}$. The resonance STMD sensitivity in the passive regime for such parameters is $\varepsilon_{\text {res }} \approx 2700 \mathrm{~V} / \mathrm{W}$ (see e.g. [17]), which is greater or comparable to the sensitivity of a typical unbiased Schottky diode [13].

We used Eq. (5) for the calculation of the output dc voltage of an STMD in the IP regime as a function of the magnitude $I_{R F}$ and frequency $f=f_{s}=\omega_{S} / 2 \pi$ of the input microwave current. These curves are shown in Figs. 6 and 7 by red dashed lines.

In order to verify the conclusions of the analytical theory of an STMD in the OOP regime we solved the LLGS Eq. 2 numerically and then numerically calculated the output dc voltage of the detector as (4). The results of our calculations are presented in Figs. 6, 7. In these figures solid blue lines and red dashed lines show the analytical dependencies of $U_{D C}$ in the OOP and IP regimes (see Eq. (21) and Eq. (5)), respectively. Dots are the results of our numerical calculations. Black crosses and green circles correspond to the cases of increasing and decreasing of the parameter (frequency $\omega_{s}$ or magnitude $I_{R F}$ of the microwave current), respectively. As one can see, the results of the analytical theory are in a reasonable agreement with the results of our numerical calculations.

As one can see from Fig. 6, in the OOP regime the STMD works as a broadband low-frequency nonresonant microwave detector and its performance is very different from the performance of an STMD operating in the traditional resonance IP regime. The response of the STMD to an input microwave current with a magnitude $I_{R F}$ is also substantially different in the cases of OOP and IP regimes of operation of an STMD (see Fig. 7). In the OOP regime, the STMD operates as a nonresonant broadband threshold microwave detector of low frequency microwave signals.

The OOP regime of operation of a STMD might be responsible for an extremely large diode volt-watt sensitivity $\varepsilon \sim 10^{5} \mathrm{~V} / \mathrm{W}$ observed in recent experiments with thermally-activated "nonadiabatic stochastic resonance" [27].

The STMD in the OOP regime could be used as a basic element of novel energy harvesting devices, in as much as it has no resonance frequency, and, therefore, could harvest energy from all the low-frequency region $\left(\omega_{s}<\omega_{H}\right)$ of the microwave spectrum. The energy conversion rate of an STMD in

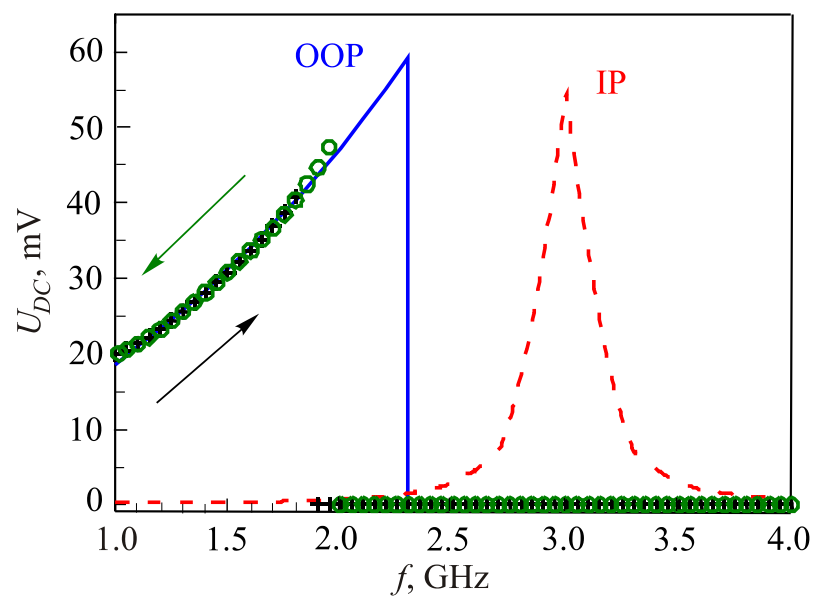

Fig. 6. (Color online) Typical dependences of the output DC voltage $U_{D C}$ on the frequency $f$ of the input microwave current $I_{R F}\left(I_{R F}=0.2 \mathrm{~mA}\right)$ for an STMD operating in the IP regime (dashed line) and OOP regime (solid line and points), respectively. Blue solid line is the analytical dependence given by Eq. (21), red dashed line is the analytical dependence given by Eq. (5). Points are the results of numerical simulations. Black crosses and green circles corresponded to the case when frequency is increased and decreased, respectively.

the OOP regime may reach $3.5 \%$ in the case $I_{R F}=I_{\mathrm{th}}\left(\omega_{s}\right)$. We believe that this ratio is sufficiently large for practical applications in the microwave energy harvesting.

\section{Conclusions}

In conclusion, we have presented a theoretical description of an STMD operating in two distinct regimes of magnetization precession. One of them is characterized by

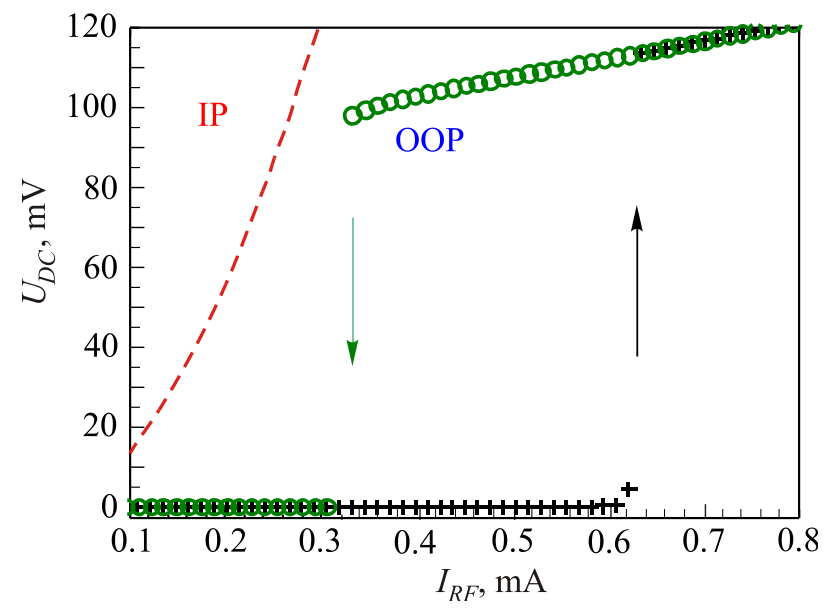

Fig. 7. (Color online) Typical dependences of the output dc voltage $U_{D C}$ on the amplitude of the input microwave current $I_{R F}(f=$ $=3 \mathrm{GHz}$ ) for an STMD operating in the IP regime (dashed line) and OOP regime (solid line and points), respectively. Blue solid line is the analytical dependence given by Eq. (21), red dashed line is the analytical dependence given by Eq. (5). Points are the results of numerical simulations. Black crosses and green circles corresponded to the case when the current is increased and decreased, respectively. 
a small-angle in-plane precession (IP regime), while the other is characterized by a large-angle out-of-plane precession (OOP regime). The performance of an STMD in these regimes is substantially different.

An STMD working in the IP regime works as a resonance-type quadratic detector having the resonance frequency close to FMR frequency of the STMD free layer. In the presence of noise this resonance-type detector can operate in two distinct sub-regimes. In one of these subregimes the detector sensitivity is limited by the magnetic noise and, therefore, noise-handling properties of the STMD in this sub-regime are different from the properties of traditional traditional semiconductor detectors. The developed formalism for the description of an STMD operating in the IP regime can be used for the optimization of the noise-handling parameters of an STMD in a wide range of temperatures.

An STMD operating in the novel OOP regime works as a threshold-type broadband detector sensitive to the microwave signals of a sufficiently low frequency and sufficiently high amplitude. We believe that the OOP regime of the STMD operation can be used for the development of novel devices for microwave energy harvesting and that the OOP regime of the STMD operation might be responsible for the extremely large diode volt-watt detector sensitivity observed in the recent experiments with "nonadiabatic stochastic resonance" [27].

Both authors would like to use this opportunity to express their deep respect and gratitude to professor Loktev, and to wish him continued success in his fruitful research activity.

This work was supported in part by the grants DMR1015175 and EPMD-1305574 from the National Science Foundation of the USA, by the Contract from the U.S. Army TARDEC, RDECOM, and by the DARPA MTO/MESO grant No.66001-11-1-4114, and by the Grant No. UU34/008 from the State Fund for Fundamental Research of Ukraine.

1. J.C. Slonczewski, J. Magn. Magn. Mater. 159, L1 (1996).

2. L. Berger, Phys. Rev. B 54, 9353 (1996).

3. J.A. Katine, F.J. Albert, R.A. Buhrman, E.B. Myers, and D.C. Ralph, Phys. Rev. Lett. 84, 3149 (2000).

4. S. Urazhdin, N.O. Birge, W.P. Pratt, Jr., and J. Bass, Phys. Rev. Lett. 91, 146803 (2003).

5. S.I. Kiselev, J.C. Sankey, I.N. Krivorotov, N.C. Emley, R.J. Schoelkopf, R.A. Buhrman, and D.C. Ralph, Nature 425, 308 (2003).

6. S. Kaka, M.R. Pufall, W.H. Rippard, T.J. Silva, S.E. Russek, and J.A. Katine, Nature 437, 389 (2005).

7. K.J. Lee, A. Deac, O. Redon, J.-P. Nozières, and B. Dieny, Nature Mater. 3, 877 (2004).

8. I.N. Krivorotov, N.C. Emley, J.C. Sankey, S.I. Kiselev, D.C. Ralph, and R.A. Buhrman, Science 307, 228 (2005).
9. F.B. Mancoff, N.D. Rizzo, B.N. Engel, and S. Tehrani, Nature 437, 393 (2005).

10. D. Houssameddine, U. Ebels, B. Delaët, B. Rodmacq, I. Firastrau, F. Ponthenier, M. Brunet, C. Thirion, J.-P. Michel, L. Prejbeanu-Buda, M.-C. Cyrille, O. Redon, and B. Dieny, Nature Mater. 6, 447 (2007).

11. A. Ruotolo, V. Cros, B. Georges, A. Dussaux, J. Grollier, C. Deranlot, R. Guillemet, K. Bouzehouane, S. Fusil, and A. Fert, Nature Nano. 4, 528 (2009).

12. A.A. Tulapurkar, Y. Suzuki, A. Fukushima, H. Kubota, H. Maehara, K. Tsunekawa, D.D. Djayaprawira, N. Watanabe, and S. Yuasa, Nature 438, 339 (2005).

13. S. Ishibashi, T. Seki, T. Nozaki, H. Kubota, S. Yakata, A. Fukushima, S. Yuasa, H. Maehara, K. Tsunekawa, D.D. Djayaprawira, and Y. Suzuki, Appl. Phys. Express 3, 073001 (2010).

14. A. Slavin, and V. Tiberkevich, IEEE Trans. Magn. 45, 1875 (2009).

15. J.C. Sankey, Y. Cui, J.Z. Sun, J.C. Slonczewski, R.A. Buhrman, and D.C. Ralph, Nature Phys. 4, 67 (2008).

16. C. Wang, Y.-T. Cui, J.Z. Sun, J.A. Katine, R.A. Buhrman, and D.C. Ralph, Phys. Rev. B 79, 224416 (2009).

17. C. Wang, Y.-T. Cui, J.Z. Sun, J.A. Katine, R.A. Buhrman, and D.C. Ralph, J. Appl. Phys. 106, 053905 (2009).

18. S. Miwa, S. Ishibashi, H. Tomita, T. Nozaki, E. Tamura, K. Ando, N. Mizuochi, T. Saruya, H. Kubota, K. Yakushiji, T. Taniguchi, H. Imamura, A. Fukushima, S. Yuasa, and Y. Suzuki, Nature Mater. 13, 50 (2014).

19. O. Prokopenko, G. Melkov, E. Bankowski, T. Meitzler, V. Tiberkevich, and A. Slavin, Appl. Phys. Lett. 99, 032507 (2011).

20. O.V. Prokopenko, I.N. Krivorotov, T.J. Meitzler, E. Bankowski, V.S. Tiberkevich, and A.N. Slavin, Spin-Torque Microwave Detectors, in: S.O. Demokritov and A.N. Slavin, Magnonics: From Fundamentals to Applications, Ser. Topics in Appl. Phys., Springer-Verlag, Berlin, Heidelberg (2013), Vol. 125.

21. D.M. Pozar, Microwave Engineering, Wiley, New York (2005).

22. A.H. MacDonald, T. Jungwirth, and M. Kasner, Phys. Rev. Lett. 81, 705 (1998).

23. C.H. Shang, J. Nowak, R. Jansen, and J.S. Moodera, Phys. Rev. B 58, R2917 (1998).

24. A.G. Gurevich and G.A. Melkov, Magnetization Oscillations and Waves, CRC, New York (1996).

25. M. Ziese and M.J. Thornton, Spin Electronics, Springer, Berlin (2001), Vol. 569.

26. O.V. Prokopenko, I.N. Krivorotov, E. Bankowski, T. Meitzler, S. Jaroch, V.S. Tiberkevich, and A.N. Slavin, J. Appl. Phys. 111, 123904 (2012).

27. X. Cheng, C.T. Boone, J. Zhu, and I.N. Krivorotov, Phys. Rev. Lett. 105, 047202 (2010). 